\title{
Segregación residencial e inserción laboral en la ciudad de Córdoba
}

Florencia Molinatti. Centro de Investigaciones y Estudios sobre Cultura y Sociedad (CIECS) - Consejo Nacional de Investigaciones Científicas y Técnicas (Conicet), Córdoba, Argentina.

RESUMEN | El presente estudio apunta a contribuir en los esfuerzos teóricos y analíticos dirigidos a investigar el impacto que tiene la segregación urbana en la calidad de la inserción de los individuos en el mercado laboral. Su punto de partida es el estudio de las transformaciones ocurridas en la mayoría de los países latinoamericanos, desde la década de 1980 en adelante, en el mercado de trabajo y en la morfología de aquellas ciudades donde dichos cambios se manifestaron. A partir de los datos de los Censos Nacionales de Población de 1991 y 2001, se indaga sobre la magnitud y evolución de la segregación residencial socioeconómica (SRS) en la ciudad de Córdoba (Argentina) y se identifican áreas homogéneas en términos de la escolaridad de los jefes de hogar. Luego se abordan de manera exploratoria, para el año 2001, los efectos de la segregación sobre la calidad de la inserción de los individuos en el mercado de trabajo, aplicando para ello un modelo de regresión logística.

PALABRAS CLAVE $\mid$ segregación, mercado de trabajo, ciudades intermedias

ABSTRACT | Based on the changes that took place in both labour market and the morphology of cities in most of Latin-American countries since the 1980s, this paper aims to contribute to the theoretical and analytical efforts regarding the impact of urban segregation on the quality of the insertion of individuals in the labour market. With data from the 1991 and 2001 National Censuses of Population, the magnitude and evolution of socioeconomic residential segregation in one intermediate city of Argentina (Córdoba) are calculated. Homogeneous areas are identified considering the education of household heads. Then, the effects of segregation on the quality of labour insertion are explored by running a logistic regression model.

KEY WORDS | segregation, labour market, intermediate cities.

Recibido el 21 de octubre de 2011, aprobado el 27 de mayo de 2012

E-mail: Florencia Molinatti, fmolinatti@hotmail.com 


\section{Introducción}

Desde hace algunos años se ha incrementado en América Latina el interés por el estudio de la segregación residencial socioeconómica (SRS). Se trata de una noción que parece evidente de por sí, en la medida en que todas las ciudades manifiestan claras señales de división social de su territorio. Sin embargo, en las últimas décadas esta tendencia ha encontrado en las ciudades latinoamericanas su mayor impulso y visibilidad, principalmente como consecuencia del notorio incremento de las desigualdades sociales y de los procesos de privatización que han tenido lugar en ellas (Kaztman, 1999; Programa de las Naciones Unidas para el Desarrollo [PNUD], 2009; Sabatini, Wormald, Sierralta \& Peters, 2007).

La mayor tradición de estudios sobre segregación residencial se encuentra en los Estados Unidos, donde se han focalizado en la dimensión étnica o racial. La utilización de esta noción aparece fuertemente en la década de 1920 con las investigaciones realizadas por los miembros de la Escuela de Chicago (Park, McKenzie, Burgess, Wirth, entre otros). El fundamento subyacente es la explicación ecológica de la sociedad y su concepto clave es la distancia social, que se contrapone con la idea de mixtura, considerada como la forma de organización ideal (Graymeyer, 1994). La segregación es entendida como el resultado de una "concentración natural" de la población urbana y determinaría la existencia de las áreas naturales de la ciudad (Park, 1999). De esta forma, los individuos buscarían agruparse por afinidades raciales, étnicas y por posición social como forma de resguardarse de los efectos fragmentadores de la personalidad generados por la aglomeración y vida en la ciudad, asociados al rápido crecimiento de las ciudades, la división del trabajo y a la difusión de la cultura urbana (Park, 1999; Simmel, 1988).

A partir de la década de 1970 se observa, principalmente en Europa, un giro teórico-metodológico e ideológico en la forma en que se aborda el fenómeno de la segregación. Estas investigaciones compartieron la preocupación por la relación entre la segregación residencial y la organización social de la economía predominante. $\mathrm{El}$ análisis de los patrones residenciales comienza a enfocarse en las estructuras sociales en lugar de las preferencias de los individuos. Sin embargo, estos estudios han sugerido lecturas empíricas e interpretaciones teóricas muy variadas, dependiendo de si la atención se centró en los principales determinantes estructurales (Harvey, Lipietz, Lojkine) o en la comprensión de los diferentes tipos de espacios en una ciudad y los procesos que producen la diferenciación social del espacio (Castells, Castells y Godard; Pinçon-Charlot et al.) (Préteceille, 1995).

En esta línea de análisis, Leal (2002) plantea que la segregación no puede considerarse únicamente como un proceso natural, tal como lo planteaba la Escuela de Chicago. La intervención pública a partir de la planificación primero, y de la política de vivienda después, afecta de forma muy directa los procesos segregativos. "La segregación social viene impulsada por las fuerzas del mercado a través de la producción y el uso del espacio urbano. Desde los planificadores urbanos hasta los promotores de vivienda, se actúa en la subdivisión y en la mercantilización del 
espacio urbano que tiende a generar procesos segregativos" (Leal, 2002, p. 60). Una de las expresiones de los procesos segregativos es la segregación residencial, es decir, el diferente comportamiento residencial de los distintos grupos sociales. Sin embargo, no se agotan en ella, ya que la segregación puede manifestarse en el uso diferencial de los medios de transporte, de los servicios públicos como la enseñanza y la salud, de los lugares de ocio y de recreación, entre otros.

Por su parte, la influencia de Max Weber llevó a incorporar la noción de distribución desigual de prestigio, reputación y poder, en el abordaje de la segregación como forma de organización del espacio físico de una ciudad. Esta influencia aparece claramente explicitada en la noción de "espacio social" de Pierre Bourdieu, quien interpreta las distancias físicas como manifestaciones de las luchas entre las clases sociales por apropiarse de la ciudad como un recurso (Ribeiro, 2003). De esta forma:

... la estructura del espacio social se manifiesta, en los contextos más diversos, en la forma de oposiciones espaciales, en las que el espacio habitado (o apropiado) funciona como una especie de simbolización espontánea del espacio social. En una sociedad jerárquica no hay espacio que no esté jerarquizado y no exprese las jerarquías y las distancias sociales, de un modo (más o menos) deformado y sobre todo enmascarado por el efecto de naturalización que entraña la inscripción duradera de las realidades sociales en el mundo natural. (Bourdieu, 1999, p. 120)

La capacidad de dominar el espacio, en especial adueñándose (material o simbólicamente) de los bienes escasos (públicos o privados) que en él se distribuyen, depende del capital poseído. Este permite mantener a distancia a personas y cosas indeseables y, al mismo tiempo, acercarse a las deseables. La proximidad en el espacio físico posibilita que la proximidad en el espacio social produzca todos sus efectos, facilitando o favoreciendo la acumulación de capital social. De la misma forma, pero a la inversa, quienes carecen de capital son mantenidos a distancia, ya sea física o simbólica, de los bienes socialmente más escasos y se los condena a codearse con las personas o bienes más indeseables y menos escasos. La falta de capital intensifica la experiencia de la finitud: encadena a un lugar (Bourdieu, 1999).

Esta última concepción, en especial la desarrollada por Bourdieu (1999) y retomada más tarde por Wacquant (2007) sobre la interacción entre el espacio físico y el espacio social, y sus repercusiones sobre el agente social y sobre su posterior posición en el espacio urbano, resulta un marco teórico interpretativo adecuado para reconocer y darles sentido a los patrones de la segregación que caracterizan las ciudades latinoamericanas. Sin embargo, no se puede desconocer el importante aporte por parte de la concepción anterior en lo referido a la cuantificación de la segregación residencial.

En América Latina, los estudios sobre la segregación residencial son mucho más recientes y la atención ha estado centrada en la dimensión socioeconómica de la segregación, considerando que las fuertes desigualdades sociales, de ingreso y de clase social representan tal vez la característica más sobresaliente de la estructura social latinoamericana. Sin embargo, autores como Rodríguez \& Arriagada (2004) 
y Sabatini, Cáceres y Cerda (2001) destacan que la investigación empírica sobre la magnitud y la evolución de la SRS para esta región es escasa, fragmentaria y poco comparable entre países. Esto podría estar asociado a la dificultad de establecer una línea divisoria, es decir, distinguir cuáles son los grupos socioeconómicos y cómo se identifican, ya que en el caso de la segregación racial la variable es mucho más sencilla de identificar y sobre sus categorías cabe poca discusión.

Partiendo de la premisa de que el "espacio/vecindario importa" (Cunha \& Jakob, 2010; Flores, 2006; Kaztman, 1999), diversas investigaciones han analizado un conjunto de riesgos o comportamientos, en un intento de evaluar el real impacto de la SRS — aquí entendida como el grado de proximidad o aglomeración espacial de estratos socioeconómicos semejantes - sobre la capacidad de respuesta a estos problemas por parte de los individuos u hogares. Este análisis se ha realizado, por ejemplo, en relación con los comportamientos de riesgo (deserción escolar, embarazo adolescente e inactividad juvenil) de niños y jóvenes (Kaztman, 1999; Sabatini et al., 2007), la vulnerabilidad social de adultos mayores (Peláez, 2006), los logros educativos de niños y adolescentes (Groisman \& Suárez, 2006; PNUD, 2009), la inserción de los individuos en el mercado de trabajo (Cunha \& Jakob, 2010; Flores, Wormald \& Sabatini, 2009; Kaztman \& Retamoso, 2005; Perelman, 2011; PNUD, 2009), entre otros.

Estas investigaciones revelan la necesidad de ampliar el marco analítico y conceptual, con el objetivo de incorporar las características contextuales en el análisis de los comportamientos individuales de las personas, en especial de aquellas que viven en zonas segregadas. Sin embargo, como resaltan los autores Kaztman y Retamoso (2005), es importante mantener una mirada crítica sobre la dirección de las líneas de la causalidad y examinar de manera pormenorizada el peso relativo de una $\mathrm{u}$ otra dirección (del contexto al individuo y del individuo al contexto).

Asimismo, algunos autores (Bourdieu, 1999; Kaztman, 1999; Wacquant, 2007) destacan el rol de la trama social del barrio en los procesos de acumulación de capital social. El barrio, por su cercanía física, se caracteriza por ser el más próximo a los espacios privados de las personas. Sin embargo, la dirección de esta incidencia varía en función de diferentes aspectos que caracterizan estos contextos residenciales, tales como el nivel de seguridad y el clima general de convivencia, el estatus social del barrio y la densidad de la trama socio-institucional. En los contextos segregados con alta concentración de la pobreza, la segregación residencial obstaculiza el proceso de formación de capital social de sus residentes, principalmente, por el distanciamiento físico y los reducidos ámbitos de interacción con otras clases. Debe considerarse, al respecto, que la formación de capital social constituye — según Katzman y Retamoso (2005) - una fuente vital de información y contactos útiles para la inserción en el mercado laboral.

El presente estudio pretende colaborar con esta línea de análisis e investigar, para el caso particular de la ciudad de Córdoba, si hay evidencias del efecto de la SRS sobre la calidad de la inserción de los individuos en el mercado de trabajo, esta última medida a partir de un indicador de informalidad laboral. Con vistas a alcanzar 
dicho objetivo, este artículo está organizado en cuatro secciones. En primer lugar, se presenta una breve caracterización del mercado laboral argentino en las últimas décadas. En segundo lugar, se describen sucintamente algunos indicadores de SRS en la ciudad para los años 1991 y 2001, y se identifican, para 2001, las zonas de segregación que se utilizarán en los posteriores análisis. En tercer lugar, para el año 2001 se evalúan, a partir de un análisis exploratorio, los diferenciales observados en términos de la calidad de la inserción en el mercado de trabajo, según características sociodemográficas, incluyendo la localización de los individuos en el espacio urbano. Por último, se desarrolla, también para el año 2001, un modelo estadístico multivariado, procurando aislar el efecto de la localización sobre la calidad de la inserción laboral.

La metodología aplicada en este trabajo es cuantitativa, con fuentes de datos secundarias. Se utilizaron los datos provenientes de los Censos Nacionales de Población realizados en 1991 y 2001 (CN1991 y CN2001) y de la Encuesta Permanente de Hogares (EPH), ondas mayo y octubre del período 1991 y 2001.

\section{Mercado de trabajo y segregación residencial: algunas cuestiones contextuales y teóricas}

\section{Las tendencias del mercado de trabajo: finales del siglo veinte y comienzos del siglo veintiuno}

A pesar de que el objetivo de este trabajo no es realizar una caracterización pormenorizada de la evolución del mercado de trabajo argentino en las últimas décadas, es necesario efectuar una breve revisión de las principales transformaciones que tuvieron lugar en la década de 1990, en especial en lo referido a la calidad de la inserción laboral de los individuos.

Hacia principios del decenio de 1970, el mercado de trabajo argentino se caracterizó por ser relativamente integrado, con alta presencia de trabajo asalariado y bajos niveles de subocupación. Sin embargo, como consecuencia del deficiente desempeño macroeconómico que experimentó la economía argentina entre mediados de 1970 y principios de 1990, esta tendencia se revirtió y el período estuvo signado por su estancamiento e inestabilidad (Altimir \& Beccaria, 1999).

Las regulaciones laborales constituyeron uno de los ingredientes clave de las reformas estructurales de los noventa. Sin embargo, cabe destacar que aquellas de mayor envergadura recién comenzaron a ser diseñadas o incluso planteadas en el periodo 1993-1994, e implementadas — al menos, parte de ellas - recién en 1995, como respuesta a la elevación a casi el $10 \%$ de la tasa de desempleo urbano en mayo de 1993. Estas medidas apuntaron a la conveniencia de adecuar la legislación referente a las relaciones individuales y colectivas con el fin de dotarla de mayor flexibilidad y, fundamentalmente, para reducir costos laborales no salariales. Estas medidas apuntaron a reducir las contribuciones patronales a la seguridad social y los costos asociados a eventos como el despido o el accidente, flexibilizar la distribución del tiempo de trabajo y descentralizar la negociación (Altimir \& Beccaria, 1999). 
Los procesos recién resumidos repercutieron en el aumento del desempleo y en la generación de inestabilidad, precariedad y baja productividad, fenómenos propios del sector informal que causaron un progresivo distanciamiento de los trabajadores con menor calificación con respecto a aquellos con mayor calificación. Al igual que en otras ciudades latinoamericanas, la nueva pobreza de la ciudad de Córdoba y sus localidades vecinas está fuertemente anclada en estos rasgos dominantes, que caracterizan a los modernos mercados de trabajo. Como se puede observar en la Figura 1, tanto las tasas de desempleo como los porcentajes de hogares pobres e indigentes, en el Aglomerado Gran Córdoba, aumentaron durante el período comprendido entre 1991 y 2001.

FIGURA 1 | Evolución de la tasa de desempleo y del porcentaje de hogares por debajo de la línea de la pobreza e indigencia. Aglomerado Gran Córdoba, 2001

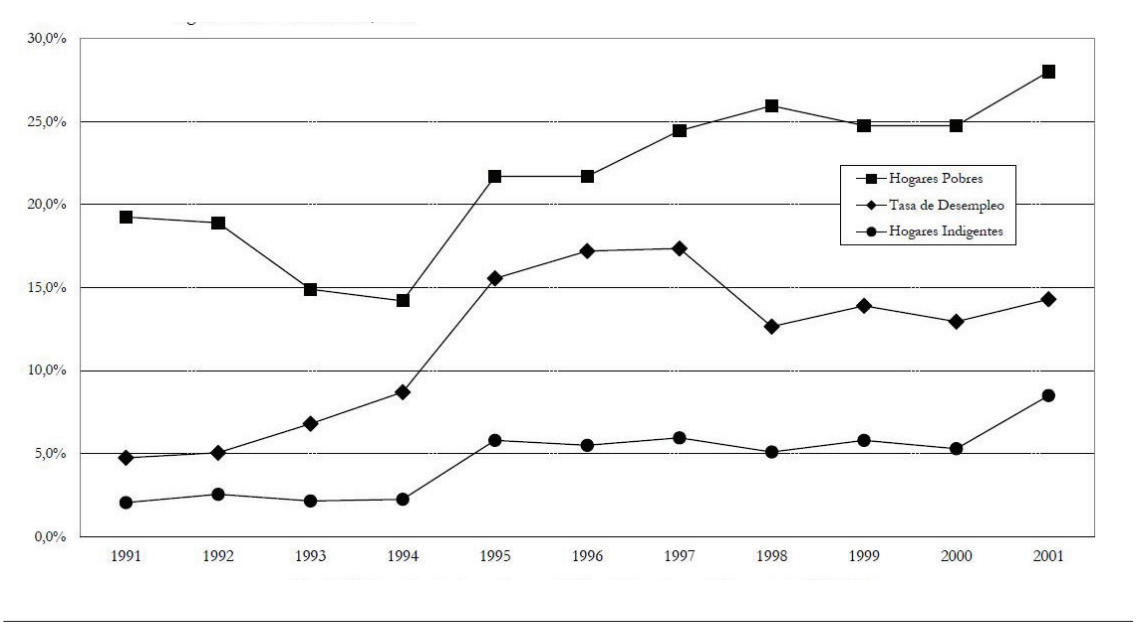

FUENTE ELABORACIÓN PROPIA CON BASE EN EPH, ONDAS MAYO Y OCTUBRE, PERIODO 1991-2001.

Este empeoramiento en las condiciones de vida estuvo acompañado por otras transformaciones en el mercado de trabajo. La tasa de participación laboral de las mujeres de catorce años o más creció significativamente en la década de 1990, subiendo del $43 \%$ en 1991 a casi el $50 \%$ en 2001. Ante la falta de dinamismo del mercado de trabajo en la generación de empleo, este aumento favoreció el alza de las tasas de desempleo, el empeoramiento de las condiciones de trabajo y la disminución de los salarios.

\section{Mercado de trabajo y segregación residencial}

Como se ha mencionado en la introducción, en América Latina existen líneas de investigación que procuran mostrar cómo la segregación residencial afecta determinadas dimensiones de la vida de los individuos y hogares. Entre los enfoques teóricos utilizados por estos estudios destacan dos: la teoría de los "efectos de barrio" (Jencks \& Mayer, 1990) y la "geografía de oportunidades" (Galster \& Killen, 1995). 
El primer abordaje subraya que si bien el barrio es concebido y distinguido como el espacio público más inmediato, a mitad de camino entre el mundo de lo privado y de lo público, la evidencia empírica de muchas ciudades muestra que la formación de áreas socialmente homogéneas en pobreza se encuentra estrechamente relacionada con la reproducción de condiciones de vida, relaciones sociales y experiencias que resultan redundantes y poco enriquecedoras (Jencks \& Mayer, 1990; Kaztman, 1999; Kaztman \& Retamoso, 2005).

El segundo abordaje busca relacionar el proceso de toma de decisiones al contexto geográfico de los individuos. Este enfoque propone que existen variaciones tanto objetivas como subjetivas en el proceso de toma de decisiones, las cuales se encuentran distribuidas desigualmente en el espacio geográfico. Dependiendo de cuán grande sea la brecha entre la estructura de oportunidades objetiva y la percepción individual, los valores, expectativas y preferencias van moldeándose negativa o positivamente, afectando la posterior toma de decisiones (Cáceres \& Sabatini, 2004; Galster \& Killen, 1995; Sierralta, 2008).

A pesar de las diferencias explicativas de estos enfoques teóricos, se observan puntos de contacto, ya que en ambos casos se explicita la importancia del lugar de residencia en la explicación de los comportamientos individuales. Entre estos abordajes se ubica el presente trabajo, que intenta responder si, una vez controladas ciertas características individuales, se encontrarán evidencias del efecto de la segregación residencial sobre la calidad de la inserción de los individuos en el mercado de trabajo.

En consecuencia, la hipótesis aquí sustentada es que la calidad de la inserción laboral, medida en este trabajo a partir de un indicador de informalidad laboral, no depende solamente de los atributos individuales, sino que también estarían influyendo en ella otros elementos de tipo contextual, como el lugar de residencia.

En relación con las variables individuales - como la edad, el sexo, el estado conyugal, el nivel educativo, la posición en el hogar, la condición migratoria- no es necesario profundizar sobre su poder explicativo, ya que se encuentra gran evidencia sobre sus efectos en la calidad de la inserción laboral en la literatura local e internacional (véase, por ejemplo, Banco Mundial-Ministerio de Trabajo, Empleo y Seguridad Social, 2008; Cerrutti \& Maguid, 2007; Perelman, 2011; Perlbach \& González, 2005, entre otros).

\section{Los procesos de segregación espacial en la ciudad de Córdoba}

\section{Aspectos conceptuales y metodológicos}

Debido a que la SRS consiste en una relación espacial con complejas conexiones con las diferencias y desigualdades sociales, es importante precisar qué se entiende por segregación y qué dimensiones distinguibles presenta.

En términos generales, "la segregación residencial consiste (...) en una relación espacial: la de separación o proximidad territorial entre personas o familias pertenecientes a un mismo grupo social, como sea que este se defina. De esta forma, el 
concepto (...) apunta a un fenómeno espacial que es independiente de la distribución del ingreso y de las diferencias sociales, por mucho que la segregación residencial esté relacionada con una y otras o que pueda incidir en ellas, por ejemplo, contribuyendo a aumentar las desigualdades" (Sabatini \& Sierralta, 2006, p. 4).

En términos particulares, se distinguen dos dimensiones objetivas de la SRS: ${ }^{1}$ la tendencia de ciertos grupos sociales a concentrarse en algunas áreas de la ciudad; y la conformación de áreas con un alto grado de homogeneidad social (Sabatini \& Sierralta, 2006, p. 4).

Estas dos dimensiones parecen ser dos formas de mirar lo mismo. Sin embargo, mientras que la primera analiza grupos sociales en términos de su localización espacial (concentración/dispersión), la segunda lo hace en términos de su composición social (homogeneidad/heterogeneidad). Por ello, cada una capta un atributo distinto de la segregación social del espacio (Sabatini \& Sierralta, 2006, p. 4).

De las dos dimensiones, la segunda es la que más claramente se asocia con problemas de desintegración social y con la formación de guetos. La homogeneidad social del espacio ayuda a aislar a un grupo social de los restantes, cuestión que se torna grave cuando se trata de grupos vulnerables o discriminados. De acuerdo con Kaztman (2001), el aislamiento social de los pobres urbanos se convierte en un obstáculo importante en la acumulación de los activos que se necesitan para dejar de ser pobres, lo que causa que la pobreza urbana aislada socialmente se constituya en un caso paradigmático de exclusión social.

La primera dimensión, el grado de concentración espacial, es capturada en este trabajo por dos índices globales: el Índice de Segregación (IS $)^{2}$ y el Índice de Disimilitud $(\mathrm{D})^{3}$. Ambos hacen referencia a la igualdad en la distribución de uno o más grupos en las zonas o en las unidades espaciales en que podemos dividir un espacio geográfico y toman como referencia los parámetros del conjunto de la ciudad (Mas-

1 La SRS también presenta una tercera dimensión, que — a diferencia de las utilizadas en este trabajo- es de carácter subjetivo. Se refiere, por un lado, a la percepción que las personas tienen del hecho de formar parte de un grupo social que tiene una forma particular de ocupar el espacio; y por otro, a la identidad y prestigio asignados a barrios o zonas completas de la ciudad, diferenciando entre barrios "malos" y barrios "exclusivos". Esta dimensión debería estudiarse con metodologías cualitativas más que cuantitativas y con estudios de caso, quedando fuera del análisis propuesto en este trabajo (Sabatini \& Sierralta, 2006, p. 5).

$2 \quad$ IS $=\frac{1}{2} \sum\left|\frac{N_{1 i}}{N_{1}}-\frac{N_{i}-N_{1 i}}{N-N_{1}}\right| ; 0 \leq I S \leq 1 ;$

donde IS es el índice de segregación; $\mathrm{N}_{1 \mathrm{i}}=$ población del grupo 1 en subdivisión territorial i-ésima; $\mathrm{N}_{1}=$ población del grupo 1 en la unidad territorial superior; $\mathrm{N}_{\mathrm{i}}$ = población total en subdivisión territorial i-ésima; $\mathrm{y}$ = población total en la unidad territorial superior (Massey \& Denton, 1988).

3

$$
\mathrm{D}=\frac{1}{2} \sum\left|\frac{N_{1 i}}{N_{1}}-\frac{N_{2 i}}{N_{2}}\right| ; 0 \leq D \leq 1
$$

donde $\mathrm{D}$ es el índice de disimilitud de Duncan; $\mathrm{N}_{1 \mathrm{i}}=$ población del grupo 1 en subdivisión territorial i-ésima; $\mathrm{N}_{2 \mathrm{i}}=$ población del grupo 2 en subdivisión territorial i-ésima; $N_{1}$ = población del grupo 1 en la unidad territorial superior; y $\mathrm{N}_{2}$ = población del grupo 2 en la unidad territorial superior (Massey \& Denton, 1988). 
sey \& Denton, 1988). La diferencia entre ambos índices consiste en que, mientras que el IS mide la distribución de un grupo respecto del total de la población de la ciudad, el D compara las proporciones de dos grupos entre sí. Ambos índices varían entre 0 (ausencia de segregación) y 1 (segregación total) y, multiplicados por cien, pueden ser interpretados como la proporción de habitantes de cada grupo que debería modificar su lugar de residencia con el fin de obtener en cada área una representación idéntica a la proporción del grupo en el conjunto urbano.

La segunda dimensión, la homogeneidad social que presentan las distintas áreas internas de la ciudad, es medida por el Índice de Aislamiento (IA $)^{4}$, que estima la probabilidad que tiene una familia de un determinado grupo, de encontrarse en el área que habita con gente de su misma condición social. Al igual que los anteriores índices, sus valores oscilan entre 0 (no aislado) y 1 (totalmente aislado). Se dice que un grupo está segregado si está mínimamente expuesto al contacto físico con los miembros de otros grupos. Este índice precisa adoptar dos supuestos teóricos para su correcta interpretación. Primero, supone que todos los contactos ocurren entre personas que habitan una misma área residencial; y segundo, que cada persona tiene la misma probabilidad de establecer contacto con cualquier otra dentro de cada área (Jargowsky, 1996).

En la lectura e interpretación de los índices de segregación es importante tener en cuenta el rol de la escala de análisis en el valor del índice. La diferente evaluación de una misma situación objetiva obedece solo al nivel de agregación escogido. Por ejemplo, si una zona de la ciudad estuviera habitada únicamente por miembros de una clase social, sería evidentemente segregada. Sin embargo, si se observa dentro de la zona, la idea de segregación carecería de sentido, ya que todos sus residentes pertenecen a la misma clase social (Rodríguez Vignoli, 2001).

$\mathrm{Al}$ ser un problema intrínsecamente metodológico, Sabatini et al. (2007) aconsejan como tratamiento la utilización de las mismas escalas geográficas de medición cuando se estudian distintas ciudades o la misma en diferentes momentos. Señalan que mientras mayor sea el tamaño del área de medición, menor será el índice, acercándose a cero cuando esta área tiende a igualarse con el área total de la ciudad; y cuanto menor sea el área de medición, mayor será el valor del índice, tendiendo al valor de uno al acercarse al tamaño mínimo posible, el del hogar. Las dimensiones objetivas de la SRS son medidas, en este trabajo, en distintas escalas espaciales: fracciones y radios censales - siendo esta última el máximo nivel de desagregación de los datos ofrecido al público por el Instituto Nacional de Estadística y Censos (INDEC) - .

El proceso de selección de la variable de segmentación socioeconómica utilizada en el análisis de la SRS tiene especial importancia, ya que, a diferencia de la segrega-

IA $=\sum_{i=1}^{n}\left(\frac{a_{i}}{A}\right) *\left(\frac{a_{i}}{t_{i}}\right) ; 0 \leq I A \leq 1$

donde IA es el índice de aislamiento; $\mathrm{a}_{\mathrm{i}}=$ población del grupo $a$ en la subdivisión territorial i-ésima; A = población del grupo $a$ en la unidad territorial superior; y $t_{\mathrm{i}}=$ población total en la subdivisión territorial i-ésima (Jargowsky, 1996). 
ción racial, identificar cuáles son los grupos socioeconómicos no es tan sencillo. En la gran mayoría de los estudios sobre SRS en América Latina se han utilizado tres tipos de variables: el primero relacionado con las necesidades básicas insatisfechas, ya sea expresadas en un índice o a partir de cada uno de sus indicadores (Marengo, 2004; Peláez, González \& Cunha, 2006; Rodríguez \& Arriagada, 2004); el segundo relacionado con la educación, ya sea del jefe de hogar o de toda la población (Kaztman, 1999; Peláez, González \& Cunha, 2006; PNUD, 2009; Rodríguez \& Arriagada, 2004); y el tercero vinculado con los ingresos a escala individual o familiar (Ariza \& Solís, 2009; Torres, 2004).

En este trabajo, al no disponerse de datos censales sobre los ingresos, se utiliza como variable proxy de la segmentación socioeconómica, el máximo nivel educativo alcanzado por el jefe de hogar, 5 agrupado en cuatro categorías:

- Bajo: sin estudios o con primario incompleto.

- Medio-Bajo: con primario completo o con secundario incompleto.

- Medio-Alto: con secundario completo o superior incompleto.

- Alto: con superior completo.

Esta decisión descansa en el supuesto de que existe una estrecha correlación entre la educación del jefe de hogar y la probabilidad de obtener mayores ingresos y una mejor calidad de vida en general. Como se observa en el Cuadro 1, el grupo de jefes de hogar de mayor nivel de instrucción percibía en 2001, en promedio, casi cuatro veces más ingresos que el grupo con menor nivel educativo. Según los resultados de la Prueba ANOVA, ${ }^{6}$ los ingresos laborales promedio de los jefes de hogar ocupados del Aglomerado Gran Córdoba difieren significativamente en las cuatro categorías educativas seleccionadas.

Además, esta elección tiene fuerte apoyo sustantivo en la creciente significación del conocimiento en las nuevas formas de producción y, por ende, en la determinación de los diferenciales en los salarios y en las protecciones sociales a las que tienen acceso trabajadores con distintos niveles de calificación (Kaztman \& Retamoso, 2005).

En el Cuadro 2 puede observarse, entre 1991 y 2001, una mejora sustancial en el nivel educativo de los jefes de hogar: todas las categorías inferiores al secundario completo disminuyen su representación tanto en términos relativos como absolutos, incrementándose correlativamente el peso de las categorías superiores. Esta evidente mejora en el nivel educativo es un fenómeno que puede responder a distintos factores, y no se debe entender mecánicamente como una movilidad social ascendente de jefes que mejoraron su nivel de instrucción. Antes bien, es probable que se explique por otros factores, tales como el recambio generacional a partir de la constitución de nuevos hogares con jefes más jóvenes y mejor instruidos que sus padres y abuelos.

5 Para evitar el efecto distorsionador de la estructura etaria sobre la educación de una población, y procurando captar a un grupo de particular influencia, se trabajó solo con los jefes y las jefas de hogar de 30 a 59 años.

6 La Prueba ANOVA (o análisis de la varianza: ANalysis Of VAriance, según terminología inglesa) es un test estadístico para probar la diferencia de medias para tres o más poblaciones. 
CUADRO 1 | Ingreso laboral promedio de los jefes de hogar ocupados según su máximo nivel educativo alcanzado. Aglomerado Gran Córdoba, 2001 (en pesos argentinos)*

\begin{tabular}{|c|c|}
\hline MÁXIMO NIVEL EDUCATIVO ALCANZADO & INGRESO LABORAL PROMEDIO \\
\hline \multicolumn{2}{|l|}{ BAJO } \\
\hline Sin estudios o con primario incompleto & $\$ 289,4$ \\
\hline \multicolumn{2}{|l|}{ MEDIO-BAJO } \\
\hline $\begin{array}{l}\text { Con primario completo o con secundario } \\
\text { incompleto }\end{array}$ & $\$ 436,4$ \\
\hline \multicolumn{2}{|l|}{ MEDIO-ALTO } \\
\hline $\begin{array}{l}\text { Con secundario completo o superior } \\
\text { incompleto }\end{array}$ & $\$ 606,2$ \\
\hline
\end{tabular}

ALTO

Con superior completo

FUENTE ELABORACIÓN PROPIA CON BASE EN EPH, ONDA OCTUBRE DE 2001. NOTA LAS DIFERENCIAS DE MEDIAS SON SIGNIFICATIVAS AL NIVEL DE $<0,01$.

* EN 2001, LA MONEDA ARGENTINA SE ENCONTRABA EN PARIDAD CON EL DÓLAR ESTADOUNIDENSE $(1$ ARS = 1 USD).

CUADRo 2 | Máximo nivel educativo alcanzado por el jefe de hogar. Ciudad de Córdoba, 1991 y 2001

\begin{tabular}{|c|c|c|c|}
\hline & 1991 & 2001 & VARIACIÓN \% \\
\hline \multirow{2}{*}{$\begin{array}{l}\text { BAJO } \\
\text { Sin estudios o con primario incompleto }\end{array}$} & 29.829 & 23.284 & \multirow{2}{*}{$-21,9$} \\
\hline & $-16 \%$ & $-11 \%$ & \\
\hline \multirow{2}{*}{$\begin{array}{l}\text { MEDIO-BAJO } \\
\text { Con primario completo o con secundario } \\
\text { incompleto }\end{array}$} & 89.355 & 84.800 & \multirow{2}{*}{$-5,1$} \\
\hline & $-47 \%$ & $-41 \%$ & \\
\hline \multirow{2}{*}{$\begin{array}{l}\text { MEDIO-ALTO } \\
\text { Con secundario completo o superior } \\
\text { incompleto }\end{array}$} & 44.148 & 61.190 & \multirow{2}{*}{38,6} \\
\hline & $-23 \%$ & $-30 \%$ & \\
\hline \multirow{2}{*}{$\begin{array}{l}\text { ALTO } \\
\text { Con superior completo }\end{array}$} & 25.189 & 36.464 & \multirow{2}{*}{44,8} \\
\hline & $-13 \%$ & $-18 \%$ & \\
\hline
\end{tabular}

FUENTE ELABORACIÓN PROPIA CON BASE EN CN1991 Y CN2001. 


\section{Segregación residencial entre 1991 y 2001 : características y evolución}

En este contexto de sustancial mejora educativa surge como interrogante cómo ha evolucionado la distribución espacial, en términos de concentración y homogeneidad, de los jefes de hogar según su máximo nivel educativo alcanzado.

Para la ciudad de Córdoba, el IS muestra que los jefes con alto nivel educativo son los que se encuentran más segregados, o menos homogéneamente distribuidos en el espacio geográfico de la ciudad; y los jefes con nivel educativo medio, los menos segregados. Por su parte, los jefes con educación baja se encuentran en una situación intermedia, aunque con valores más cercanos a los calculados para los jefes con alto nivel educativo. Dicho patrón parece no tener diferencias significativas según las escalas geográficas de medición y se asemeja al encontrado en otras ciudades latinoamericanas (Cuadro 3).

CUADRo 3 | Dimensión 1: Índice de Segregación, según máximo nivel educativo alcanzado por el jefe de hogar, por radio y fracción censal. Ciudad de Córdoba, 1991 y 2001

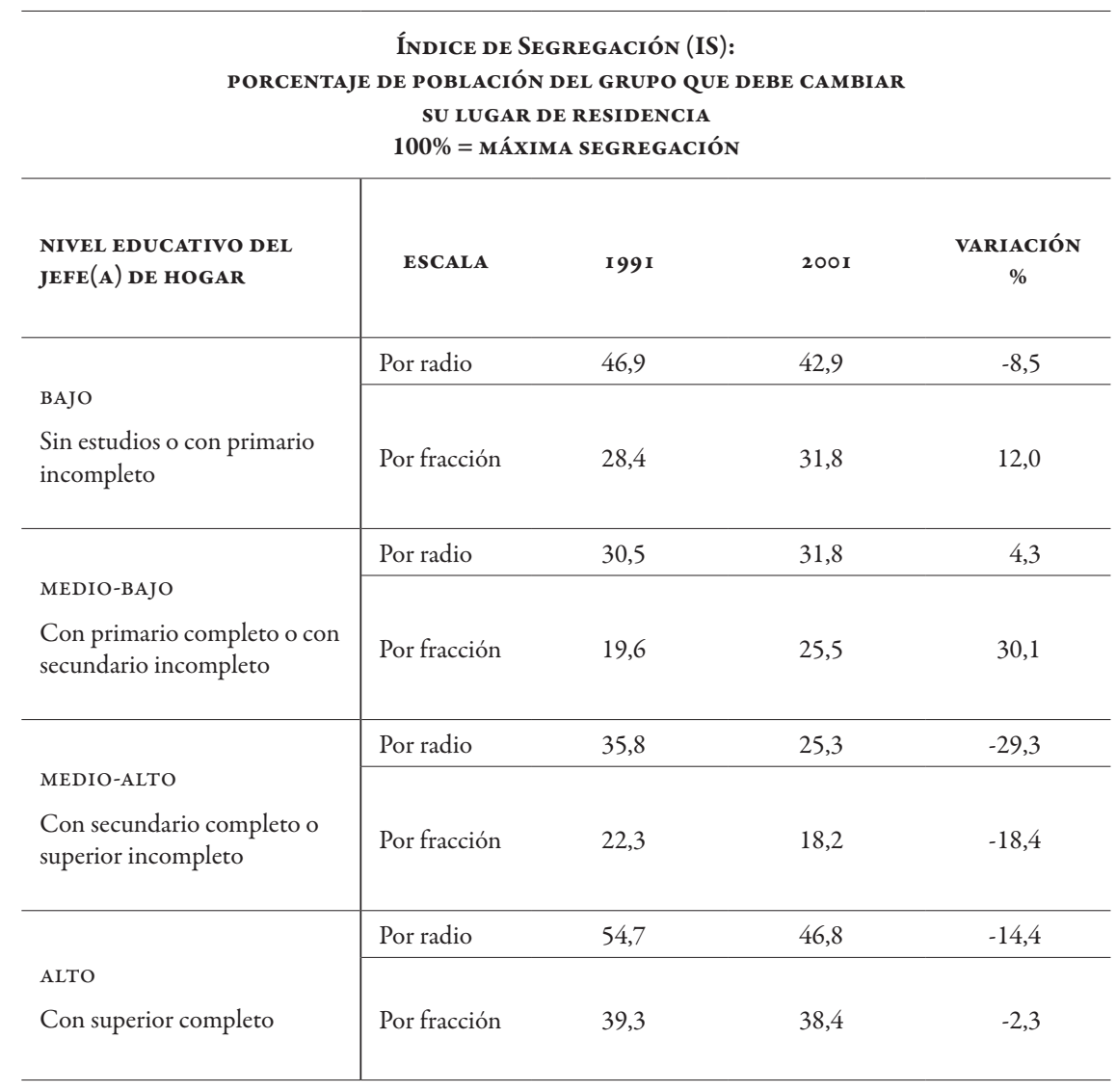

FUENTE ELABORACIÓN PROPIA CON BASE EN CN1991 Y CN2001. 
Entre 1991 y 2001, la segregación de los jefes con alto nivel educativo retrocedió significativamente y lo hizo con más fuerza a nivel de radio censal, donde era más fuerte. En relación con el grupo de jefes con educación baja, se observa un comportamiento ambiguo de la segregación: disminuye su concentración a nivel de radio censal, pero aumenta en la escala geográfica superior.

Son particularmente notables las variaciones de los grupos intermedios: aumenta, aunque levemente a escala de radio censal, la segregación del grupo con educación media-baja, mientras disminuye significativamente la del grupo con educación media-alta, en ambas escalas.

Mientras este índice ofrece información sobre el grado relativo de concentración de cada uno de los grupos con respecto al resto de la ciudad, el D permite evaluar la distribución de los grupos de a pares. Por ejemplo, mientras el IS para el grupo de jefes con educación baja permite medir cuán segregado está este grupo en relación con el resto de la ciudad, el $\mathrm{D}$, para este mismo grupo, indica cuán segregado se encuentra con respecto a cada uno de los otros grupos de jefes.

Como puede observarse en el Cuadro 4, el D entre grupos muestra una estrecha correlación entre nivel educativo del jefe(a) de hogar y la ocupación del espacio, sugiriendo una alta correspondencia entre la distribución espacial de los grupos y las distancias educativas existentes entre ellos. El índice alcanza valores más bajos al calcularse entre grupos educativos contiguos, y valores más altos entre grupos salteados y extremos.

Entre 1991 y 2001 se observa que mientras a nivel de radio censal la segregación retrocedió para todos los grupos con respecto a cada uno de los otros, a nivel de fracción censal la segregación aumentó para todos, excepto la correspondiente a los grupos bajo y medio-bajo entre sí. Esto indica un cambio de escala en los patrones de la dimensión 1 de la segregación residencial, que comienza a actuar con más fuerza en las escalas superiores.

Es particularmente notable que, a pesar de la disminución entre 1991 y 2001 de la segregación entre los extremos, el índice todavía registra valores muy altos para ambas escalas geográficas de medición; por ejemplo, más del $70 \%$ de los jefes con educación alta (o baja) debería cambiar su lugar de residencia (radio censal) para obtener una distribución homogénea en toda la ciudad. A pesar de que estos valores son similares a los observados entre negros y blancos en las áreas metropolitanas más segregadas de los Estados Unidos en 2000, ${ }^{7}$ tal comparación debe ser tomada con muchas reservas, principalmente por las diferencias en el tamaño de escalas utilizadas (los sectores censales - census tract - norteamericanos son una unidad espacial intermedia entre los radios y las fracciones censales aquí considerados) y por la dimensión de la segregación medida (racial en los Estados Unidos y socioeconómica en la Argentina).

Tradicionalmente, los estudios sobre segregación residencial han tendido a focalizar su atención en la primera dimensión de la segregación, privilegiando los índices que dan cuenta del grado de concentración de los grupos sociales en el espacio

7 http://www.censusscope.org/us/rank_dissimilarity_white_black.html 
geográfico. A partir de una revisión de los trabajos sobre segregación, Sabatini y Sierralta (2006) observan una tendencia a omitir la segunda dimensión, tendencia justificada en la alta correlación $(r=0,827)$ constatada por Glaeser para las ciudades estadounidenses entre ambas dimensiones de la segregación.

CUADRO 4 | Dimensión 1: Índice de Disimilitud, según máximo nivel educativo alcanzado por el jefe de hogar, por radio y fracción censal. Ciudad de Córdoba, 1991 y 2001

\begin{tabular}{|c|c|c|c|c|c|c|}
\hline \multicolumn{7}{|c|}{$\begin{array}{l}\text { ÍNDICE DE Disimilitud }(D) \text { : } \\
\text { PORCENTAJE DE POBLACIÓN DEL GRUPO QUE DEBE CAMBIAR SU LUGAR DE RESIDENCIA } \\
100 \%=\text { MÁXIMA SEGREGACIÓN }\end{array}$} \\
\hline \multicolumn{3}{|c|}{ Nivel educativo del jefe(a) de hogar } & \multirow{2}{*}{ Escala } & \multirow{2}{*}{1991} & \multirow{2}{*}{2001} & \multirow{2}{*}{ Variación \% } \\
\hline Grupo 1 & Grupo 2 & Relación 1-2 & & & & \\
\hline \multirow{2}{*}{ Bajo } & \multirow{2}{*}{ Medio-Bajo } & \multirow{2}{*}{ Contiguos } & Por radio & 36,2 & 26,9 & $-25,7$ \\
\hline & & & Por fracción & 17,1 & 16,3 & $-4,7$ \\
\hline \multirow{2}{*}{ Medio-Bajo } & \multirow{2}{*}{ Medio-Alto } & \multirow{2}{*}{ Contiguos } & Por radio & 40,0 & 34,9 & $-12,8$ \\
\hline & & & Por fracción & 26,7 & 27,1 & 1,5 \\
\hline \multirow{2}{*}{ Medio-Alto } & \multirow{2}{*}{ Alto } & \multirow{2}{*}{ Contiguos } & Por radio & 36,5 & 28,1 & $-23,0$ \\
\hline & & & Por fracción & 20,8 & 21,5 & 3,4 \\
\hline \multirow{2}{*}{ Bajo } & \multirow{2}{*}{ Medio-Alto } & \multirow{2}{*}{ Salteados } & Por radio & 62,7 & 54,7 & $-12,8$ \\
\hline & & & Por fracción & 40,5 & 41,0 & 1,2 \\
\hline \multirow{2}{*}{ Medio-Bajo } & \multirow{2}{*}{ Alto } & \multirow{2}{*}{ Salteados } & Por radio & 60,8 & 56,7 & $-6,7$ \\
\hline & & & Por fracción & 44,1 & 46,6 & 5,7 \\
\hline \multirow{2}{*}{ Alto } & \multirow{2}{*}{ Bajo } & \multirow{2}{*}{ Extremos } & Por radio & 76,5 & 70,8 & $-7,5$ \\
\hline & & & Por fracción & 56,2 & 57,3 & 2,0 \\
\hline
\end{tabular}

FUENTE ELABORACIÓN PROPIA CON BASE EN CN1991 y CN2001.

Sin embargo, para el caso latinoamericano esto no es válido, ya que una alta segregación por concentración espacial no se encuentra necesariamente correlacionada con una alta segregación por homogeneidad social. Por ejemplo, de acuerdo con Sabatini et al. (2007), las elites presentan una alta segregación en la dimensión 1 , pero una relativamente baja segregación en la dimensión 2 . A pesar de que las elites latinoamericanas se encuentran altamente concentradas en lo que puede denominarse "cono de alta renta", comparten dicho cono con todos los grupos sociales. Por esta razón, es importante observar cómo ha evolucionado la segregación por homogeneidad en la década de los noventa en la ciudad de Córdoba. 
Si se observan ahora las cifras del IA, que mide la probabilidad de que un individuo (u hogar) comparta la unidad espacial con un individuo (u hogar) de su mismo grupo, ellas también sugieren que hay SRS en Córdoba, pero de bastante menor intensidad que la observada en la dimensión anterior (Cuadro 5). En la ciudad de Córdoba, entre 1991 y 2001, se observa, para ambas escalas, una disminución del aislamiento de los jefes de hogar con educación baja y media-baja y un aumento, aunque de menor significancia, del aislamiento de los jefes con educación igual o superior a la educación media completa. Sin embargo, sin negar la presencia de segregación por homogeneidad social, estos datos tienen que ser tomados con cautela. Es probable que este hecho se explique, principalmente, por el aumento absoluto y relativo de la cantidad de jefes con nivel de instrucción medio-alto y alto ocurrido en el período analizado.

CUADRO 5 | Dimensión 2: Índice de Aislamiento, según máximo nivel educativo alcanzado por el jefe de hogar, por radio y fracción censal. Ciudad de Córdoba, 1991 y 2001

ÍNDice de Aislamiento (IA):

EXPOSICIÓN DEL GRUPO $A$ AL CONTACTO CON MIEMBROS DEL MISMO GRUPO.

100\% = MÁXIMO AISLAMIENTO Y MAYOR SEGREGACIÓN

\begin{tabular}{|c|c|c|c|c|}
\hline Nivel educativo del jefe(a) de hogar & Escala & 1991 & 2001 & $\begin{array}{c}\text { Variación } \\
\%\end{array}$ \\
\hline \multirow{2}{*}{$\begin{array}{l}\text { ВАјо } \\
\text { Sin estudios o con primario incompleto }\end{array}$} & Por radio & 31,0 & 21,6 & $-30,3$ \\
\hline & Por fracción & 21,1 & 16,3 & $-22,7$ \\
\hline \multirow{2}{*}{$\begin{array}{l}\text { MEDIO-BAJO } \\
\text { Con primario completo o con } \\
\text { secundario incompleto }\end{array}$} & Por radio & 54,8 & 49,0 & $-10,6$ \\
\hline & Por fracción & 50,5 & 46,6 & $-7,7$ \\
\hline \multirow{2}{*}{$\begin{array}{l}\text { MEDIO-ALTO } \\
\text { Con secundario completo o superior } \\
\text { incompleto }\end{array}$} & Por radio & 33,6 & 35,0 & 4,2 \\
\hline & Por fracción & 27,2 & 32,4 & 19,1 \\
\hline \multirow{2}{*}{$\begin{array}{l}\text { ALTO } \\
\text { Con superior completo }\end{array}$} & Por radio & 32,4 & 33,6 & 3,7 \\
\hline & Por fracción & 23,0 & 28,6 & 24,3 \\
\hline
\end{tabular}

FUENTE ELABORACIÓN PROPIA CON BASE EN CN1991 y CN2001. 
Estos cambios observados en la década de 1990 en los patrones de la segregación residencial, tanto por concentración espacial como por homogeneidad social, pueden estar relacionados con dos tendencias, también identificadas para el Área Metropolitana de Santiago de Chile por investigadores del Instituto de Estudios Urbanos y Regionales de la Pontificia Universidad Católica de Chile (Cáceres \& Sabatini, 2004; Sabatini, et al., 2007): la dispersión espacial de las elites y la aproximación física de los pobres a las elites. ${ }^{8}$

En lo referido a la dispersión de las elites, se observa el surgimiento de un nuevo mercado inmobiliario, con gran capacidad de transformación de la estructura urbana. Este cambio se hace visible en el desarrollo de grandes proyectos inmobiliarios tanto de tipo residencial (countries, barrios cerrados, barrios de lujo en altura), como de tipo comercial (shoppings y grandes supermercados), que impulsan la salida de las familias de elites, en especial las jóvenes, del tradicional cono de alta renta hacia zonas ubicadas en la periferia de la ciudad. El diseño (barrio cerrado, seguridad privada) y el gran tamaño de estos proyectos facilitan la inserción de las familias de elite en zonas ocupadas por grupos sociales con menor poder adquisitivo.

La segunda tendencia, la aproximación física de los pobres a las áreas de residencia de los grupos con mayor poder adquisitivo, posiblemente está relacionada con la precarización del empleo y con el debilitamiento del Estado de Bienestar que tuvo lugar en la década de 1990. En este contexto, la geografía de oportunidades y la búsqueda de espacios de diversidad social adquieren especial relevancia en el espacio geográfico de la ciudad. Por ejemplo, un cuarto de las "villas miseria" que surgieron entre 1992 y 2001 en la ciudad de Córdoba se ubicaron próximas al área central o al cono de alta renta (Buthet, Baima de Borri \& Calvo, 2007).

\section{Identificación de las zonas de segregación}

Las medidas recién utilizadas proporcionan una información sintética y global de la segregación en la ciudad de Córdoba en el período 1991-2001, y permiten conocer el grado de segregación de la ciudad como un todo. Sin embargo, no posibilitan la identificación de las áreas segregadas en el espacio geográfico de la ciudad. Para esto es necesaria la utilización de medidas georreferenciadas, que tengan como punto de partida la semejanza (o no) de las unidades espaciales vecinas. En este trabajo se optó por una medida basada en la autocorrelación espacial, el Índice de Moran Local.

El Índice de Moran es un indicador de asociación espacial que evalúa la existencia de conglomerados (clusters) en la distribución espacial de una determinada variable, el cual puede ser calculado de forma localizada para cada unidad espacial (Local)

8 Para América Latina, Portes (1989) analiza cómo el crecimiento desordenado de las ciudades a mediados del siglo XX generó un patrón de segregación residencial en donde los sectores de bajos ingresos se encontraban relativamente cerca de las clases pudientes. Las invasiones de tierras de las poblaciones de bajos y medianos recursos compensaban las tendencias de dispersión y segregación por clase social. Sin embargo, la apertura del mercado del período neoliberal cambió los patrones de segregación social, principalmente por la proliferación de comunidades cerradas en la mayoría de las ciudades. Estas urbanizaciones, con rejas y muros, hicieron posible la proximidad de los sectores de ingresos altos y medios a los pobres. No obstante, el crecimiento de las áreas metropolitanas también contribuyó a aislar a los asentamientos más pobres de la periferia (Roberts \& Portes, 2008). 
o como medida general (Global). Mientras la versión global del índice ofrece un estadístico que permite establecer el grado de aglomeración de unidades semejantes, la versión local posibilita la creación de un mapa en el que se pueden detectar las unidades espaciales (en este caso, los radios censales del Censo 2001) que se encuentran rodeadas por otras semejantes o no (Anselin, 1995; Cunha \& Jacob, 2010).

Considerando la variable "años promedio de educación de los jefes de hogar de 30 a 59 años", se clasificaron ${ }^{9}$ los radios censales en cinco grupos (Figura 2):

- Alto-Alto: radios con jefes con educación alta rodeados de otros con condición semejante.

- Bajo-Bajo: radios con jefes con educación baja rodeados de otros con condición semejante.

- Alto-Bajo: radios con jefes con educación alta rodeados de otros con jefes con educación baja.

- Bajo-Alto: radios con jefes con educación baja rodeados de otros con jefes con educación alta.

- No significativo: radios con gran heterogeneidad.

FIGURA 2 Resultados del Índice de Moran Global para los años promedio de educación de los jefes de hogar, según radios censales. Ciudad de Córdoba, 2001

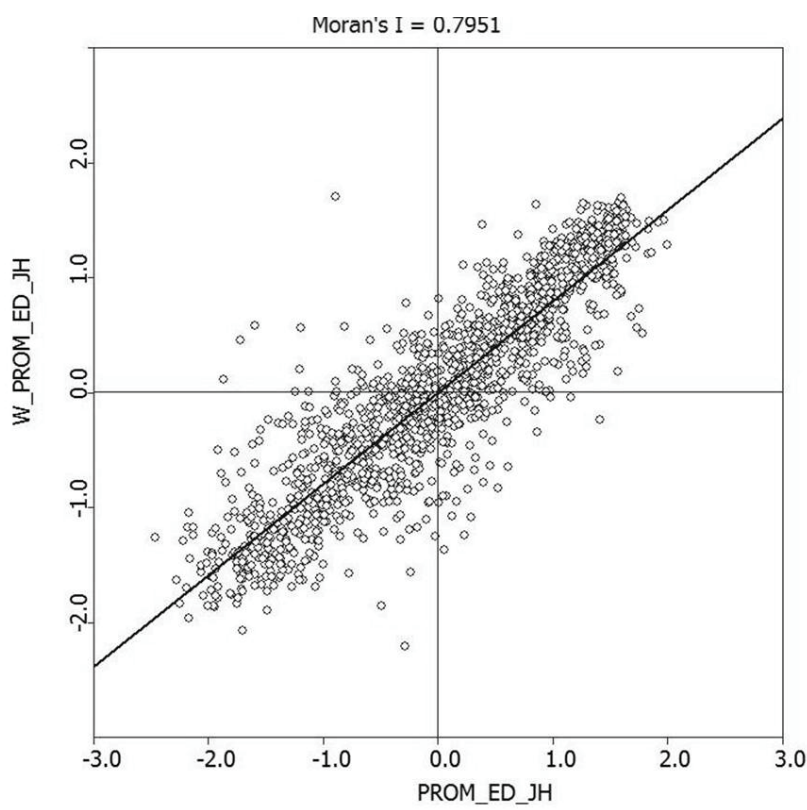

FUENTE ELABORACIÓN PROPIA CON BASE EN CN2001.

Nota P-Seudo significativo al Nivel de 0,001, 999 permutaciones.

9 Esta clasificación fue obtenida a partir de la aplicación del Índice de Moran Local en el software libre GeoDa (versión 9.8.14). 
Como se observa en el scatter plot de Moran (Figura 2), el análisis de la distribución espacial de los jefes de hogar según los años promedio de educación mostró una fuerte autocorrelación espacial positiva $(\mathrm{I}=0,7951, \mathrm{p}<=0,001)$. La mayoría de las asociaciones son entre radios con similares características en cuanto a la educación del jefe de hogar (cuadrante I y II del gráfico).

En la Figura 3 se presenta el mapa creado a partir de la aplicación del Índice de Moran Local a nivel de radio censal según el promedio de educación del jefe de hogar. En correspondencia con la fuerte autocorrelación espacial positiva detectada, en el mapa se observa la existencia de fuertes concentraciones de población en la ciudad de Córdoba, lo cual muestra que la segregación residencial no se registra solamente para la población más pobre. Mientras la periferia, en especial la ubicada en el sur y este de la ciudad, presenta una gran concentración de jefes de hogar con baja educación (Bajo-Bajo), en la zona central y noroeste se observa una mayor predominancia de jefes de hogar con mayor educación (Alto-Alto).

FIGURA 3 | Resultados del Índice de Moran Local para los años promedio de educación de los jefes de hogar, según radios censales. Ciudad de Córdoba, 2001

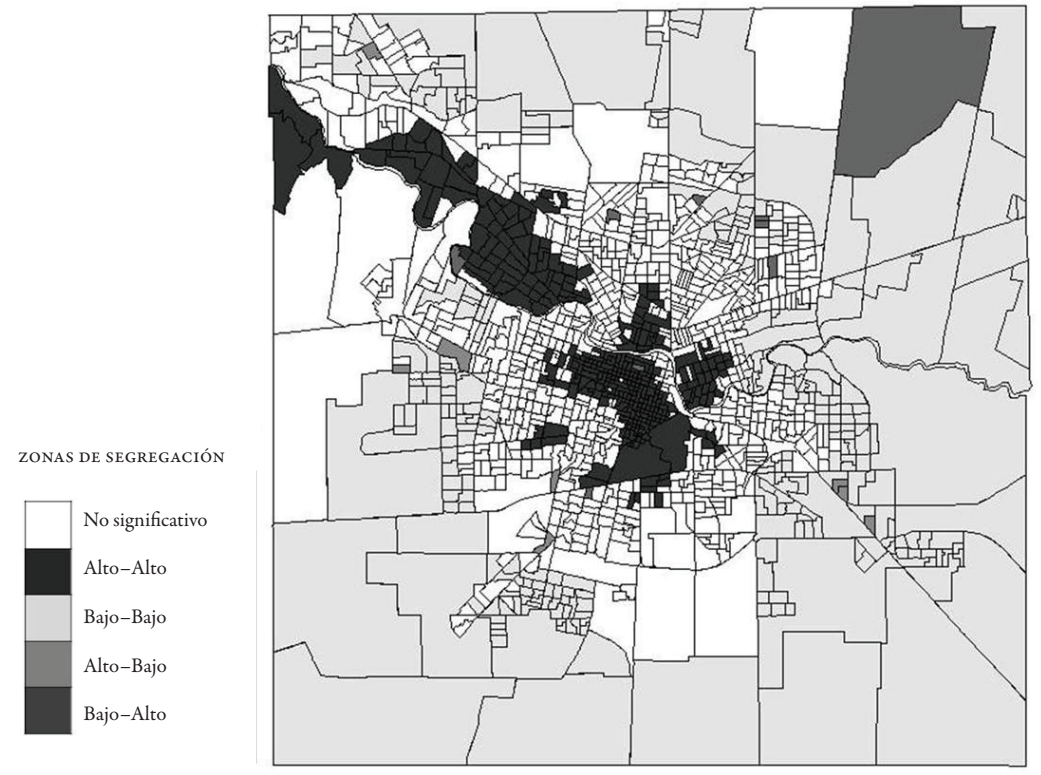

FUENTE ELABORACIÓN PROPIA CON BASE EN CN2001.

\section{Resultados: los efectos de la segregación residencial sobre la calidad de la inserción laboral}

Siempre que se plantean relaciones entre las características contextuales (barrios segregados) y características individuales (por ejemplo, informalidad laboral), es 
conveniente mantener una mirada crítica sobre la dirección de las líneas de la causalidad y medir de manera pormenorizada el peso de una u otra dirección. Según Kaztman y Retamoso (2005), una de las líneas de causalidad más examinadas es la que asigna una mayor probabilidad de fijar su residencia en los barrios más pobres a los que tienen mayores problemas en el mercado laboral. Es decir, que la concentración de las personas con baja calificación en ciertos vecindarios es interpretada como una consecuencia de lo que les pasa a las personas en el mercado de trabajo.

Sin embargo, aun admitiendo que las oportunidades de empleo son la causa de los desplazamientos hacia las áreas más pobres y del deterioro de sus antiguos residentes, también cabe reconocer que una vez que las personas se establecen o permanecen en esos barrios de pobreza más concentrada y homogénea, sus eventuales vínculos con el mercado de trabajo van a ser afectados por factores y procesos propios del entorno. Esta línea de causalidad se fundamenta en los efectos del contexto de residencia sobre las personas que habitan en zonas segregadas, en especial sobre los niños y adolescentes socializados en esas zonas, para los cuales la influencia del barrio claramente antecede al proceso de inserción laboral.

El análisis aquí presentado consta de dos etapas. La primera comprende un abordaje exploratorio, en el que se evalúan los diferenciales observados en términos de la calidad de la inserción al mercado de trabajo, aquí medida a partir de un indicador de informalidad laboral, ${ }^{10}$ según características sociodemográficas, incluida la posición relativa en el espacio geográfico de la ciudad. En la segunda se formula un modelo multivariado, con el objetivo de aislar el efecto de la segregación sobre la informalidad laboral, una vez controladas las características individuales.

A partir de la literatura disponible, se seleccionaron potenciales variables explicativas. En el Cuadro 6 se resumen los resultados del análisis descriptivo del indicador de informalidad laboral para la población económicamente activa ocupada (PEAO) de 18 a 64 años, ${ }^{11}$ censada en 2001 en la ciudad de Córdoba.

Puede observarse que, según el sexo de la persona, en 2001 los hombres presentan una menor informalidad laboral que las mujeres. En cuanto a la educación, se percibe que a medida que aumenta el nivel educativo del individuo, disminuye su probabilidad de encontrarse en una ocupación informal: mientras 8 de cada 10 personas con educación baja se encuentran informalmente ocupadas, solo 2 de cada 10 personas con educación alta se encuentran en esa misma situación.

10 Dentro de la categoría "ocupados informales" se incluye a los siguientes grupos: los asalariados que no poseen aportes jubilatorios; los asalariados no profesionales que trabajan en establecimientos con menos de cinco ocupados; los trabajadores por cuenta propia no profesionales; los trabajadores familiares sin salario; y el servicio doméstico. La idea subyacente a esta clasificación es captar relaciones laborales precarias, dadas por la utilización de recursos propios, con escaso o nulo acceso al crédito, escasa disponibilidad de bienes de capital y utilización de mano de obra familiar. La tecnología empleada solo permite desarrollar actividades de baja calificación, con insumos de baja calidad, derivando en la producción de bienes y servicios que únicamente permiten la subsistencia del empleado y su grupo familiar. Es decir, este abordaje de informalidad está más cerca de una medición de calidad del empleo (precariedad laboral), que de un enfoque meramente legal (Perlbach \& González, 2005).

11 Para los fines analíticos de este trabajo, solo se trabajó con población que reside en viviendas particulares, excluyendo del análisis a las personas que viven en instituciones colectivas o en la calle. También se excluyó del análisis a los asalariados y trabajadores por cuenta propia que no pudieron ser clasificados según su condición de informalidad laboral, por faltar datos sobre la calificación de la actividad que realizan. 
CUADro 6 | Condición de informalidad laboral de la PEAO de 18 a 64 años, según características sociodemográficas. Ciudad de Córdoba, 2001

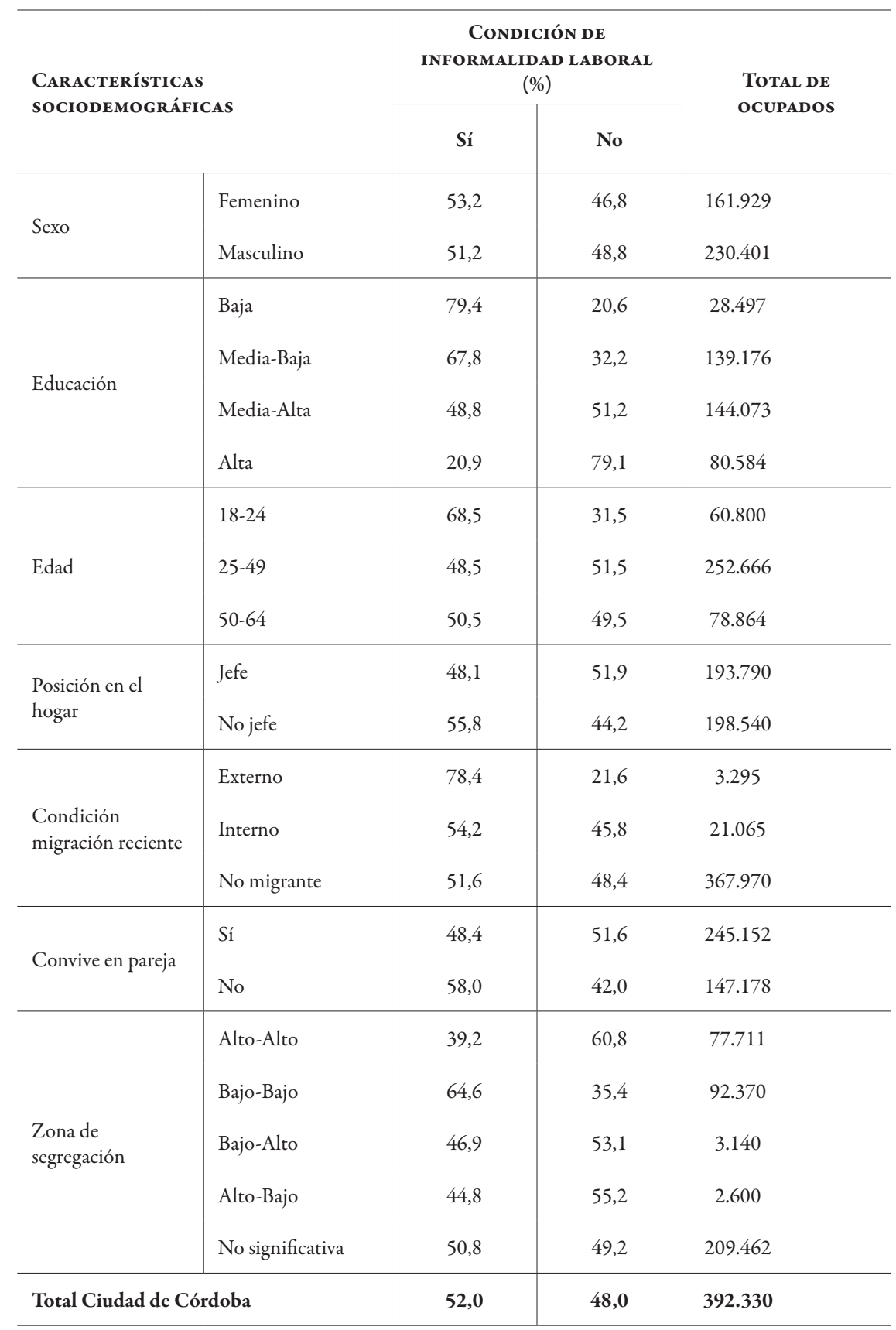

FUENTE ELABORACIÓN PROPIA CON BASE EN CN2001 
La edad de la persona también influye en la condición de informalidad laboral. La curva que relaciona el porcentaje de ocupados informales con la edad tiene una forma de "u", ${ }^{12}$ indicando que existen mayores probabilidades de tener una ocupación informal para aquellos trabajadores jóvenes y los de mayor edad. En el Cuadro 5 se observa que el grupo de edad con menor porcentaje de ocupados informales es el de ocupados de 25 a 49 años.

En relación con la posición del individuo en el hogar, se observa que los jefes, en comparación al resto de los miembros del hogar, tienen menor probabilidad de encontrarse ocupados informalmente. También influye sobre esa condición la convivencia o no en pareja de la persona ocupada: entre los ocupados que no conviven en pareja, el porcentaje de ocupados informales es casi 10 puntos porcentuales superior al que se observa entre los ocupados que sí conviven en pareja.

Los datos muestran que si se analiza la informalidad laboral según la condición migratoria reciente del ocupado, se observa que es mayor en el caso de los migrantes externos que en el de los migrantes internos o no migrantes: alrededor del 77\% de los migrantes externos se caracteriza por tener una ocupación informal, cifra casi 30 puntos porcentuales superior al promedio de la ciudad.

Finalmente, la localización relativa de la persona en el espacio geográfico de la ciudad, según las zonas de segregación identificadas en el punto anterior, parece estar fuertemente relacionada con la calidad de la inserción en el mercado de trabajo, ya que mientras casi el $40 \%$ de los ocupados que residen en zonas con baja concentración de jefes de hogar con bajo nivel educativo (Alto-Alto) tiene una ocupación informal, ese porcentaje asciende a $65 \%$ en las zonas con alta concentración de este grupo de jefes (Bajo-Bajo).

Un resultado interesante es el que surge de la comparación entre las zonas clasificadas como Bajo-Bajo y Bajo-Alto, es decir, entre aquellas con mayor homogeneidad y concentración de jefes con educación inferior al secundario completo, y aquellas donde los jefes con bajo nivel educativo se encuentran próximos a jefes con mejor nivel educativo. Como puede observarse en el Cuadro 5, los residentes en zonas con menor homogeneidad (Bajo-Alto) tienen menor probabilidad de encontrarse ocupados informalmente, que sus pares que residen en áreas muy homogéneas.

Sin embargo, debe reconocerse que estas asociaciones encontradas no pueden tratarse como indicaciones inequívocas del efecto de la segregación residencial sobre la calidad de la inserción laboral, medida aquí en términos de informalidad, ya que depende de la dirección de causalidad. Responder a esta disyuntiva no es tarea fácil, en especial por la limitación de las fuentes de datos tradicionales, que no permiten realizar estudios de tipo longitudinal o profundizar sobre los efectos de la localización en la inserción de los individuos en el mercado de trabajo. Sin embargo, la aplicación de modelos estadísticos multivariados permite una aproximación al aislar el efecto de la segregación residencial sobre la informalidad laboral, una vez controlados los efectos de las demás variables. El modelo aquí empleado es la regresión logística, que tiene como objetivo principal producir un modelo de predicción de probabilidades o riesgos a partir de variables explicativas. En este caso se intenta

Datos no presentados en este trabajo. 
predecir la probabilidad de ocurrencia de un evento (informalidad laboral) a partir de la ocurrencia de otros factores: sexo, edad, educación, posición en el hogar, convivencia en pareja, condición de migración reciente y localización residencial.

Los parámetros estadísticos fueron estimados a partir de la utilización del paquete estadístico SPSS (Statistical Package for the Social Sciences). Las variables independientes y sus categorías utilizadas en el modelo ${ }^{13}$ son:

- Sexo: masculino y femenino (control).

- Edad: entre 18 y 24 años y entre 25 y 64 años (control).

- Educación: inferior al secundario completo y secundario completo o más (control).

- Posición en el hogar: jefe de hogar (control) y no jefe de hogar.

- Convive en pareja: sí (control) y no.

- Condición migratoria reciente: migrante externo, migrante interno y no migrante (control).

- Zona de segregación: alta-alta, baja-baja, alta-baja, baja-alta y no significativa (control).

Como se observa en el Cuadro 7, los efectos de las variables sociodemográficas se muestran coherentes con los encontrados en otras investigaciones: mayor probabilidad de tener una ocupación informal para las personas de sexo femenino, jóvenes (menores de 25 años), con bajo nivel educativo (inferior al secundario completo), no jefes, que no conviven en pareja y que son migrantes, en especial externos.

Específicamente, en lo que se refiere a la localización de los individuos en el espacio interno de la ciudad, el modelo muestra que residir en áreas clasificadas como Alto-Alto (áreas homogéneas con alta concentración de jefes de hogar con educación alta) disminuye en un 18\% el riesgo de tener una ocupación informal. En cambio, si el individuo reside en áreas clasificadas como Bajo-Bajo (áreas homogéneas con alta concentración de jefes de hogar con educación baja), el riesgo aumenta en un $26 \%$. Las áreas categorizadas como Bajo-Alto no presentan efectos significativos sobre la informalidad laboral, sugiriendo que residir en áreas más heterogéneas tiene efectos positivos en la calidad de la inserción laboral de los jefes con educación baja, disminuyendo su propensión a la informalidad laboral.

En resumen, considerando los resultados del modelo, se puede decir que, incluso controlando variables importantes y claves (como el sexo, la edad y la educación), el lugar de residencia del individuo parece asumir un papel importante en la calidad de su inserción en el mercado de trabajo. Más aún, los ocupados que residen en áreas con alta concentración de jefes con bajo nivel educativo pero próximas a áreas con jefes con mejor condición educativa presentan menos riesgo de tener una ocupación informal que aquellos que residen en áreas también con alta concentración de jefes con educación baja, pero más homogéneas. Esto indica que la informalidad

13 Este modelo responde por $67 \%$ de las personas ocupadas de 18 a 64 años, con una especificidad del $65,9 \%$ y una sensibilidad del $67,9 \%$, siendo, por lo tanto, un modelo aceptable. El punto de corte utilizado fue del $50 \%$, valor bastante próximo al observado en la población $(52 \%)$ 
laboral no es solamente una cuestión de pobreza o educación, sino que el contexto de residencia ejerce importantes influencias en la calidad de la inserción del individuos en el mercado de trabajo.

CUADRO 7 | Riesgo de tener una ocupación laboral informal. Resultados de regresión logística. Ciudad de Córdoba, 2001

\begin{tabular}{|c|c|c|c|c|c|c|c|c|}
\hline \multirow{2}{*}{\multicolumn{2}{|c|}{$\begin{array}{l}\text { CARACTERÍSTICAS } \\
\text { SOCIODEMOGRÁFICAS }\end{array}$}} & \multirow{2}{*}{ B } & \multirow{2}{*}{ E.T. } & \multirow{2}{*}{ WALD } & \multirow{2}{*}{ Sig. } & \multirow{2}{*}{$\begin{array}{r}\text { EXP } \\
(B)\end{array}$} & \multicolumn{2}{|c|}{ I.C. $95 \%$ PARA EXP(B) } \\
\hline & & & & & & & 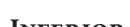 & \\
\hline Sexo & Femenino &, 188 & 008 & 566,080 &, 000 & 1,207 & 1,189 & 1,226 \\
\hline Edad & 18 a 24 años &, 615 &, 011 & 3137,060 &, 000 & 1,849 & 1,809 & 1,889 \\
\hline Educación & $\begin{array}{l}\text { Educación } \\
\text { Baja }\end{array}$ & 1,324 & ,008 & 29406,520 &, 000 & 3,759 & 3,702 & 3,816 \\
\hline $\begin{array}{l}\text { Posición en } \\
\text { el hogar }\end{array}$ & No jefe & , 183 & ,008 & 520,741 &, 000 & 1,200 & 1,182 & 1,219 \\
\hline $\begin{array}{l}\text { Convive en } \\
\text { pareja }\end{array}$ & No & ,331 & ,008 & 1800,371 & ,000 & 1,392 & 1,371 & 1,413 \\
\hline Condición & $\begin{array}{l}\text { Migrante } \\
\text { interno }\end{array}$ & ,238 & ,015 & 235,271 & ,000 & 1,268 & 1,230 & 1,307 \\
\hline reciente & $\begin{array}{l}\text { Migrante } \\
\text { externo }\end{array}$ & 1,528 & ,044 & 1197,152 & ,000 & 4,607 & 4,225 & 5,023 \\
\hline \multirow{4}{*}{$\begin{array}{l}\text { Zona de } \\
\text { segregación }\end{array}$} & Alto-Alto &,- 203 & ,009 & 475,308 &, 000 &, 816 &, 802 &, 831 \\
\hline & Bajo-Bajo & ,229 & ,009 & 666,872 &, 000 & 1,257 & 1,235 & 1,279 \\
\hline & Bajo-Alto &, 014 &, 038 & ,139 &, 709 & 1,014 & 941 & 1,094 \\
\hline & Alto-Bajo &,- 142 &, 042 & 11,487 &, 001 & 867 & ,799 & 942 \\
\hline \multicolumn{2}{|l|}{ Constante } &,- 901 & ,007 & 14474,471 &, 000 & ,406 & & \\
\hline
\end{tabular}

Variable dependiente: Condición de informalidad laboral

FUENTE ELABORACIÓN PROPIA CON BASE EN CN2001. 


\section{Consideraciones finales}

Los resultados alcanzados en este trabajo ponen de manifiesto dos complejidades: por un lado, la vinculada al propio fenómeno de la SRS, fundamentalmente en lo que concierne a la dificultad para establecer la línea divisoria entre los grupos socioeconómicos y las dimensiones de la segregación; y por otro, la relacionada con la identificación y cuantificación del efecto que la localización residencial del individuo tiene sobre sus comportamientos, sus vulnerabilidades y condiciones generales de vida.

En cuanto a la existencia o no de SRS en la ciudad de Córdoba, las medidas calculadas en este trabajo sugieren la existencia de segregación en ella en el período considerado. Se observa una estrecha correlación entre el nivel educativo alcanzado por el jefe de hogar y su ocupación del espacio geográfico. Los grupos extremos (jefes con educación baja y alta) son los que presentan mayor segregación por concentración (dimensión 1). Estos resultados son similares a los encontrados en otras ciudades latinoamericanas. En lo referido a la segunda dimensión de la SRS (homogeneidad social), los resultados indican que en el patrón de segregación latinoamericano, una alta segregación por concentración no se traduce necesariamente en una alta segregación por homogeneidad social.

La evolución de la segregación entre 1991 y 2001 en la ciudad de Córdoba es ambigua. Los jefes con educación superior se encuentran menos concentrados en el espacio geográfico de la ciudad, pero más aislados. Lo mismo sucede con los jefes que se encuentran en el otro extremo de la distribución, es decir, los jefes con educación inferior a la primaria completa. El valor del D para estos grupos externos sugiere que, a pesar de los retrocesos observados, todavía se encuentran muy desigualmente distribuidos en la ciudad: alrededor del $70 \%$ de los jefes con educación alta (o baja) deberían cambiar su lugar de residencia para obtener una distribución homogénea en la ciudad.

En lo referido a los efectos del lugar de residencia sobre la calidad de la inserción laboral, los resultados aquí presentados, aunque todavía exploratorios, sugieren la existencia de segmentación laboral, la cual se ha visto intensificada durante el período analizado. Durante la década de 1990 se observó un aumento de las tasas de desocupación y de la proporción de hogares con pobreza e indigencia por ingresos. Además, se constató un empeoramiento de las condiciones laborales, siendo que el porcentaje de asalariados no registrados en el sistema de previsión social aumentó un $22 \%$ en este período.

Los cambios por los que pasó el mercado de trabajo en la ciudad de Córdoba en particular, y en Argentina en general, a lo largo de la década de los noventa, resultaron en transformaciones económicas, sociales e institucionales que alteraron el perfil de la mano de obra. Estos procesos alcanzaron de forma socialmente negativa y con mayor intensidad a la población más pobre, especialmente a los menos escolarizados y calificados. En este trabajo se observó que existen variaciones significativas en la probabilidad de tener una ocupación informal conforme al lugar de residencia, aun cuando son controlados los atributos individuales de los ocupados. 
Esto implica que las personas que residen en áreas con alta homogeneidad y concentración de jefes de hogar con educación baja (zonas de segregación Bajo-Bajo) tienen una menor probabilidad de tener una ocupación formal.

Un resultado interesante es el obtenido para aquellas zonas de residencia de población poco escolarizada que se encuentran próximas a las áreas habitadas por los estratos con el mejor promedio educativo de la ciudad (categoría Bajo-Alto). En este caso, las estimaciones muestran un menor riesgo de caer en la informalidad laboral para los habitantes de esas zonas en comparación con los residentes en zonas con alta segregación (Bajo-Bajo). Estas diferencias en torno a la inserción en el mercado de trabajo formal pueden ser analizadas en términos de capital social, ya que la cercanía física de los hogares pobres a aquellos con mayores activos facilita la circulación de información sobre oportunidades laborales. Estos resultados también han sido encontrados en trabajos realizados en Campinas, Brasil (Cunha \& Jacob, 2010) y en Santiago de Chile (Sabatini et al., 2007).

Los resultados expuestos estarían apoyando las líneas explicativas que afirman que las diferencias encontradas entre los residentes de distintos barrios deberían atribuirse no solo a los atributos individuales, sino también a la influencia de los contextos que socializan a los vecinos o limitan sus oportunidades en forma diferenciada. Retomando la noción "efectos de lugar" de Bourdieu (1999), la ausencia o escasez de algunas de las formas de capital (humano, económico, social) de los individuos y hogares que residen en contextos segregados en términos de pobreza, los encadena a un lugar, reduciendo sus espacios de interacción, limitando sus contactos a aquellas personas de su misma condición y, como consecuencia, reforzando su situación de pobreza y vulnerabilidad y reproduciendo en una especie de círculo vicioso sus condiciones de vida.

Sin negar la potencialidad explicativa de las líneas de investigación que vinculan las características individuales de los sujetos con sus condiciones laborales, en este trabajo se plantea la necesidad de incorporar las características contextuales en el análisis de los comportamientos individuales, en especial por lo que esto significaría en términos de políticas públicas. El dilema crucial para las políticas públicas es contemplar o no la heterogeneidad que presentan las ciudades. El principal argumento a favor de la adopción de estrategias territoriales en la formulación e implementación de políticas públicas está ligado a la existencia de fuertes externalidades relacionadas con la residencia en barrios con alta concentración de pobres. En estas áreas, la probabilidad de conseguir un trabajo formal es menor, porque existe una baja proporción de personas empleadas en el sector formal, reduciendo las posibilidades de algunos de conseguir trabajo por medio de su red de relaciones sociales (Torres, 2005). OEURE 


\section{Referencias bibliográficas}

Altimir, O. \& Beccaria, L. (1999). El mercado de trabajo bajo el nuevo régimen económico en Argentina. LC/L. 1217, Serie Reformas Económicas N² 28. Santiago de Chile: Comisión Económica para América Latina y el Caribe (Cepal). En http://www.eclac.cl/publicaciones/ $\mathrm{xml} / 0 / 4550 / \mathrm{lcl} 1217 \mathrm{e} . \mathrm{pdf}$

Anselin, L. (1995). Local indicators of spatial association - LISA. Geographical Analysis, 2(27), 93 115. doi: 10.1111/j.1538-4632.1995.tb00338.x

Ariza, M. \& Solís, P. (2004). Dinámica socioeconómica y segregación espacial en tres áreas metropolitanas de México, 1990 y 2000. Estudios Sociológicos, 27(79), 171-209. En http://redalyc. uaemex.mx/src/inicio/ArtPdfRed.jsp?iCve=59820689006

Banco Mundial/Ministerio de Trabajo, Empleo y Seguridad Social, Argentina (2008). Aportes a una nueva visión de la informalidad laboral en la Argentina. Buenos Aires: Banco Mundial/ Ministerio de Trabajo, Empleo y Seguridad Social. En http://cdi.mecon.gov.ar/doc/bm/ argentina/2.pdf

Bourdieu, P. (1999). Efectos de lugar. En P. Bourdieu (Ed.), La miseria del mundo (pp. 119-124). Madrid: Fondo de Cultura Económica. Artículo en http://es.scribd.com/doc/87407773/ Bourdieu-Efectos-de-Lugar

Buthet, C., Baima de Borri, M. \& Calvo, D. (2007). La evolución de las villas de emergencia de la ciudad de Córdoba 2001-2007: localización y estimación de población. Córdoba: Servicio Habitacional y de Acción Social.

Cáceres, G. \& Sabatini F. (2004). Barrios cerrados en Santiago de Chile: Entre la exclusión y la integración residencial. Santiago de Chile: Lincoln Institute of Land Policy/Instituto de Geografía de la Pontificia Universidad Católica de Chile.

Cerrutti, M. \& Maguid, A. (2007). Inserción laboral e ingresos de los migrantes de países limítrofes y peruanos en el gran Buenos Aires. Notas de Población, 83, 75-98. En http://www.eclac.cl/ publicaciones/xml/9/30029/lcg2340-P3.pdf

Cunha, J. M. P. \& Jakob, A. A. E. (2010). Segregação socioespacial e inserção no mercado de trabalho na Região Metropolitana de Campinas. Revista Brasileira de Estudos de População, 27(1), 115-139. http://dx.doi.org/10.1590/S0102-30982010000100008

Flores, C. (2006). Conseqüências da segregação residencial: teoria e métodos. En J. M. P. Cunha (Ed.). Novas metrópoles paulistas: população, vulnerabilidade e segregação (pp. 197-230). Campinas: Nepo/Unicamp. En http://www.nepo.unicamp.br/textos/publicacoes/livros/vulnerabilidade/arquuivos/arquuivos/vulnerab_cap_7_197_230.pdf

Flores, C., Wormald, G. \& Sabatini, F. (2009). Segregación residencial y trayectorias laborales de jefes de hogar en conjuntos de vivienda social en Chile. Trabajo presentado en el XXVIII Congress of the Latin American Studies Association (LASA 2009): Rethinking Inequalities, Rio de Janeiro, 11 al 14 de junio.

Galster, G. \& Killen S. (1995). The geography of metropolitan opportunity: A reconnaissance and conceptual framework. Housing Policy Debate, 6(1), 7-43. doi: 10.1080/10511482.1995. 9521180

Grafmeyer, Y. (1994). Regards sociologiques sur la ségrégation. En J. Brun \& C. Rhein (Eds.), La ségrégation dans la ville. Concepts et mesures (pp. 85-117). Paris: L'Harmattan. 
Groisman, F. \& Suárez, A. L. (2006). Segregación residencial en la Ciudad de Buenos Aires. Población de Buenos Aires, 3(4), 27-37. En http://www.redligare.org/IMG/pdf/segregacion_residencial_ciudad_buenos_aires.pdf

Jargowsky, P. (1996). Take the money and run: Economic segregation in U.S. Metropolitan Areas. American Sociological Review, 61(6), 984-998. URL estable: http://www.jstor.org/stable/2096304

Jencks, Ch. \& Mayer S. (1990). The social consequences of growing up in a poor neighborhood. En L. Lynn \& M. Mcgeary (Comps.), Inner-City Poverty in the United States (pp. 111-186). Washington, D.C.: Committee on National Urban Policy, National Research Council.

Kaztman, R. (1999). El vecindario también importa. En R. Kaztman (Ed.), Activos y estructuras de oportunidades de estudios sobre las raices de la vulnerabilidad social en Uruguay (pp. 263307). Montevideo: Comisión Económica para América Latina y el Caribe (Cepal)/Programa de Naciones Unidas para el Desarrollo (PNUD).

Kaztman, R. (2005). Segregación residencial en Montevideo: Desafíos para la equidad educativa. Documento presentado en la Reunión de Expertos sobre Población y Pobreza en América Latina y el Caribe, Centro Latinoamericano de Demografía (Celade), Santiago de Chile: 14 y 15 de noviembre. En http://www.cepal.cl/celade/noticias/paginas/5/27255/Kaztman_Retamoso.pdf

Kaztman, R. \& Retamoso A. (2005). Segregación espacial, empleo y pobreza en Montevideo. Revista de la CEPAL, 85, 131-148. En http://www.cepal.cl/publicaciones/xml/8/21048/ $\operatorname{lcg} 2266 \mathrm{eKaztmanRetamoso.pdf}$

Leal, J. (2002). Segregación social y mercados de vivienda en las grandes ciudades. RES. Revista Española de Sociologia, 2, 59-75. En http://www.fes-web.org/uploads/files/res/res02/04.pdf

Marengo, C. (2004). Una aproximación a la segregación residencial, como punto de partida en la formulación de políticas. Revista INVI, 19(50), 165-181. En http://www.revistas.uchile.cl/ index.php/INVI/article/viewFile/8813/8620

Massey, D. \& Denton N. (1988). The dimensions of residential segregation. Social Forces, 67(2), 281315. URL estable: http://www.jstor.org/stable/2579183

Park, R. E. (1999). La ciudad como laboratorio social. En R. E. Park, La ciudad y otros ensayos de ecología humana (E. Martínez, Trad., pp. 115-126). Barcelona: Ediciones del Serbal.

Peláez, E. (2006). Selectividad residencial de los adultos mayores en la ciudad de Córdoba. Astrolabio, 3 (noviembre). En http://www.astrolabio.unc.edu.ar/articulos/sociedadadultosmayores/ articulos/pelaez.php

Peláez, E., González, L. \& Cunha, J. M. P. (2006). Dimensiones de la segregación residencial en el Gran Córdoba (Argentina) y su comparación con RM de Campinas (Brasil). Carta económica regional, 20(100), 31-47.

Perelman, L. (2011). Diferencias socioespaciales e inserción laboral en la Ciudad de Buenos Aires. (Cuaderno de Trabajo de Cedem N $\left.{ }^{\circ} 11\right)$. Buenos Aires: Dirección General de Estadística y Censos, Ministerio de Hacienda, Gobierno de la Ciudad Autónoma de Buenos Aires (GCBA).

Perlbach, I. \& González, R. (2005). Informalidad en el mercado laboral argentino: un modelo de probabilidad de ocurrencia. Trabajo presentado en el VII Congreso Nacional de Estudios de Trabajo, Buenos Aires, 10 al 12 de agosto.

Portes, A. (1989). Latin American urbanization in the years of the crisis. Latin American Research Review, 24(3), 7-44. URL estable: http://www.jstor.org/stable/2503697 
Préteceille, E. (1995). Ségrégations urbaines. Sociétés Contemporaines, 22(22-23), 5-14. URL estable: http://www.persee.fr/web/revues/home/prescript/article/socco_1150-1944_1995_ num_22_1_1528

Programa de las Naciones Unidas para el Desarrollo (PNUD). (2009). Segregación residencial en Argentina. Colección Aportes para el Desarrollo Humano en Argentina/2009. Buenos Aires: Autor. En http://hdr.undp.org/en/nhdr/support/other/national/pnud_libro2.pdf

Ribeiro, L. C. (2003). Segregação residencial e políticas públicas: Análise do espaço social da cidade na gestão do território. En E. Rassi Neto \& C. M. Bógus (Eds.), Saúde nos aglomerados urbanos: uma visão integrada (pp. 155-180). Brasilia: Organização Pan-Americana da Saúde. En http://hdl.handle.net/10071/3406

Roberts, B. \& Portes, A. (2008). Conclusión. Enfrentando la ciudad del libre mercado. La acción colectiva urbana en América Latina, 1980-2000. En: A. Portes, B. Roberts \& A. Grimson (Eds.), Ciudades latinoamericanas. Un análisis comparativo en el umbral del nuevo siglo (pp. 425-464). Buenos Aires: Prometro Libros. Versión PDF en http://rimd.reduaz.mx/coleccion_desarrollo_migracion/ciudades_latinoamericanas/c7.pdf

Rodríguez, J. \& Arriagada, C. (2004). Segregación residencial en la ciudad latinoamericana. EURE, 30(89), 5-24. doi: 10.4067/S0250-71612004008900001

Rodríguez Vignoli, J. (2001). Segregación residencial socioeconómica: ¿qué es?, ¿cómo se mide?, ¿qué está pasando?, ¿importa? LC/L.1576-P, Serie Población y Desarrollo No 16. Santiago de Chile: Comisión Económica para América Latina y el Caribe (Cepal)/Centro Latinoamericano de Demografía (Celade). En http://www.eclac.cl/publicaciones/xml/8/7888/lcl1576-P. pdf

Sabatini, F., Cáceres, G. \& Cerda, J. (2001). Segregación residencial en las principales ciudades chilenas: Tendencias de las tres últimas décadas y posibles cursos de acción. EURE, 27(82), 21-42. En http://www.eure.cl/wp-content/uploads/2001/12/EURE_82_02_CACERES.pdf

Sabatini, F. \& Sierralta, C. (2006). Medición de la segregación residencial: Meandros teóricos y metodológicos, y especificidad latinoamericana. Documento de Trabajo № 38. Santiago de Chile: Instituto de Estudios Urbanos y Territoriales, Pontificia Universidad Católica de Chile.

Sabatini, F., Wormald, G., Sierralta, C. \& Peters, P. (2007). Segregación residencial en Santiago: Tendencias 1992-2002 y efectos vinculados con su escala geográfica. Documento de Trabajo $\mathrm{N}^{\mathrm{o}}$ 37. Santiago de Chile: Instituto de Estudios Urbanos y Territoriales, Pontificia Universidad Católica de Chile.

Sierralta, C. (2008). Efectos de la segregación residencial socioeconómica en los jóvenes de extracción popular en Santiago de Chile (1992-2002). Tesis de Maestría para optar título de Magíster en Desarrollo Urbano, Instituto de Estudios Urbanos y Territoriales, Pontificia Universidad Católica de Chile, Santiago de Chile. En http://www.ieut.cl/wp-content/uploads/2011/01/ EfectosdelasegregacinresidencialsocioeconmicaenlosjvenespobresdeSantiagodeChile1992-2002.pdf

Simmel, G. (1988). La metrópoli y la vida mental. En M. Bassols, R. Donoso, A. Massolo \& A. Méndez (Comps.), Antología de sociología urbana (pp. 47-61). México, D.F.: Universidad Nacional Autónoma de México (UNAM).

Torres, H. (2004). Segregação residencial e políticas públicas: São Paulo na década de 1990. Revista Brasileira de Ciências Sociais, 19(54), 41-55. En http://redalyc.uaemex.mx/src/inicio/ ArtPdfRed.jsp?iCve=10705403 
Torres, H. (2005). Políticas sociais e território: uma abordagem metropolitana. En E. Marques \& H. Torres (Eds.), São Paulo: segregação, pobreza e desigualdades sociais (pp. 297-320). São Paulo: Editora Senac.

Wacquant, L. (2007). Los condenados de la ciudad. Gueto, periferias y Estado. Buenos Aires: Siglo Veintiuno Editores Argentina. 